\title{
Root causes of intraoperative hypoglycemia: a case series.
}

Eric S. Schwenk

Thomas Jefferson University

Boris Mraovic

Thomas Jefferson University

Ryan P. Maxwell

Thomas Jefferson University

Gina S. Kim

Thomas Jefferson University

Jesse M. Ehrenfeld

Massachusetts General Hospital

Follow this and additional works at: https://jdc.jefferson.edu/anfp

See next page for additional authors

Part of the Anesthesiology Commons

Let us know how access to this document benefits you

\section{Recommended Citation}

Schwenk, Eric S.; Mraovic, Boris; Maxwell, Ryan P.; Kim, Gina S.; Ehrenfeld, Jesse M.; and Epstein, Richard H., "Root causes of intraoperative hypoglycemia: a case series." (2012). Department of Anesthesiology Faculty Papers. Paper 20.

https://jdc.jefferson.edu/anfp/20

This Article is brought to you for free and open access by the Jefferson Digital Commons. The Jefferson Digital Commons is a service of Thomas Jefferson University's Center for Teaching and Learning (CTL). The Commons is a showcase for Jefferson books and journals, peer-reviewed scholarly publications, unique historical collections from the University archives, and teaching tools. The Jefferson Digital Commons allows researchers and interested readers anywhere in the world to learn about and keep up to date with Jefferson scholarship. This article has been accepted for inclusion in Department of Anesthesiology Faculty Papers by an authorized administrator of the Jefferson Digital Commons. For more information, please contact: JeffersonDigitalCommons@jefferson.edu. 


\section{Authors}

Eric S. Schwenk, Boris Mraovic, Ryan P. Maxwell, Gina S. Kim, Jesse M. Ehrenfeld, and Richard H. Epstein 


\title{
As submitted to: \\ Journal of Clinical Anesthesia
}

And later published as:

\section{Title: Root Causes of Intraoperative Hypoglycemia: A Case Series}

\author{
Volume 24, Issue 8, December 2012, Pages 625-630. \\ http://dx.doi.org/10.1016/j.jclinane.2012.04.009
}

\section{Corresponding author:}

Eric S. Schwenk, MD

Resident, Department of Anesthesiology

Suite 8290

Thomas Jefferson University Hospital

111 South $11^{\text {th }}$ Street

Philadelphia, PA 19107

Co-authors:

Boris Mraovic, MD

Associate Professor of Anesthesiology

Suite 8280

Thomas Jefferson University Hospital

111 South $11^{\text {th }}$ Street

Philadelphia, PA 19107

Ryan P. Maxwell, DO

Resident, Department of Anesthesiology

Suite 8290

Thomas Jefferson University Hospital

111 South $11^{\text {th }}$ Street

Philadelphia, PA 19107

Gina S. Kim, BS

Medical student, Jefferson Medical College

Thomas Jefferson University Hospital

111 South $11^{\text {th }}$ Street

Philadelphia, PA 19107 
Jesse M. Ehrenfeld, MD, MPH

Assistant Professor of Anesthesiology and Biomedical Informatics

Massachusetts General Hospital

Boston, MA

Current address:

Department of Anesthesiology

Vanderbilt University Medical Center

Room 701

Medical Arts Building

121121 st Ave

Nashville, TN 37212

Richard H. Epstein, MD, CPHIMS

Professor of Anesthesiology

111 South $11^{\text {th }}$ Street

Suite 6215F, Gibbon Building

Philadelphia, PA 19107

Funding: this study was funded entirely through department funds

Conflicts of Interest: there are no conflicts of interest to report

Keywords: hypoglycemia, intraoperative, human error, insulin, shock, diabetes 


\section{Abstract}

Study Objective: To describe the root causes of intraoperative hypoglycemic events.

Design: Retrospective analysis.

Setting: Large academic teaching hospital.

Patients: 80,379 surgical patients, American Society of Anesthesiologists Physical Status $1-5$.

Interventions: None.

Measurements: Blood glucose values, insulin and oral hypoglycemic medication doses, and doses of glucose or other medications for hypoglycemia treatment.

Main results: Hypoglycemia in many patients had multiple etiologies, and many (8 of 17) involved preventable errors. The most common were ineffective communication, circulatory shock, failure to monitor, and excessive insulin.

Conclusion: Intraoperative hypoglycemia was rare but often preventable. Better communication among providers and from providers to patients could potentially reduce the number of events. Many transient episodes of hypoglycemia did not result in any 
apparent complications, making their clinical importance uncertain. Critically ill patients in circulatory shock represent a group that may require close glucose monitoring.

\section{Introduction}

Although well described in other clinical areas, the circumstances associated with the development of intraoperative hypoglycemia (blood glucose (BG) $\leq 40 \mathrm{mg} / \mathrm{dL}$ ) are not as clear. Clinical data are needed to understand the relevant issues in this environment.

The paucity of intraoperative data on hypoglycemia reflects difficult clinical recognition and a low frequency of intraoperative BG monitoring. Autonomic responses are difficult to interpret due to hemodynamic variability during surgery and are blunted by anesthetics and beta-blocker therapy [1], and patients are unable to report symptoms during general anesthesia. Surgical stress increases the BG [2], potentially decreasing concern by anesthesia care providers about the need to monitor intraoperatively to detect hypoglycemia. The performance of numerous concurrent tasks during intraoperative care and the multi-step procedure required for accurate point-of-care (POC) monitoring may also affect sample frequency. In addition, concerns regarding the accuracy of POC glucose meters in the intraoperative setting [3] and lack of provider certification may lead to decreased use. Finally, since few patients have arterial catheters or convenient exposed 
sites from which venous blood may be sampled to send to the central lab, glucose monitoring may be omitted.

We present a series of 17 cases of intraoperative hypoglycemia that occurred during a five-year period. Attention is focused on human factors that lead to potentially avoidable events.

\section{Materials and Methods}

\section{Study Design and Data Collection}

We conducted a retrospective database study, approved by the Institutional Review Board of Thomas Jefferson University with waiver of informed patient consent, of 80,379 surgical cases from 10/2005 to 12/2010 at a large, academic hospital during which at least one intraoperative BG was measured. The intraoperative period was defined as the interval when the patient entered until he or she left the operating room. As an initial screen, the anesthesia information management system (AIMS) database was queried to identify patients where a BG of $\leq 40 \mathrm{mg} / \mathrm{dL}$ was documented in the intraoperative record, either by manual entry (for POC measurements), or electronic entry (for central lab measurements). For the root cause analysis, patient charts and electronic medical records were reviewed in detail by at least two investigators to identify potentially spurious values. Of the 18 patients with an intraoperative $\mathrm{BG} \leq 40 \mathrm{mg} / \mathrm{dL}$, one was excluded due to a transcription error (POC value 13 instead of 130), confirmed by checking the central POC database to which the devices transmit data when docked in the 
charging stations. The remaining 17 patients' paper and electronic records underwent a root cause analysis by at least two investigators to establish the cause(s) of the hypoglycemic event.

The following data were recorded from the perioperative period: timing and method of BG measurements; timing, type, and dose of insulin and any oral hypoglycemic agents; and timing and dose of any glucose treatments. After analysis, root causes were agreed upon by a consensus of the research group. The hospital has an intraoperative glucose management protocol for patients receiving care in an intensive care unit, but use is explicitly restricted to those locations. Although some anesthesia care providers follow the protocol in the OR, such use is not a departmental policy; thus, we did not assess adherence to that particular protocol.

\section{Hospital Protocols}

For any patient receiving insulin or other diabetic medications, BG must be checked a minimum of every six hours. If a patient is treated for hypoglycemia $(\mathrm{BG} \leq 40$ $\mathrm{mg} / \mathrm{dL}$ ), a recheck of the $\mathrm{BG}$ must be performed within 15 minutes.

Insulin infusions in the ICU setting are recommended for patients with two consecutive BGs > $180 \mathrm{mg} / \mathrm{dL}$. The insulin infusion monitoring protocol mandates hourly BG checks while an infusion is running until the BG is between 140 and $180 \mathrm{mg} / \mathrm{dL}$ and stable for two consecutive hours. If that requirement is met and no change in clinical status occurs, the frequency of BG checks can be decreased to every two hours. The department of anesthesiology does not endorse any specific insulin protocol for the intraoperative setting, however. 


\section{Glucose Measurements}

Glucose values were determined with a Beckman ${ }^{\circledR}$ LX-20 chemistry analyzer (Beckman Coulter, Brea, California), located in the central chemistry lab, or Accu-Chek ${ }^{\circledR}$ Inform glucose POC meters (Roche Pharmaceuticals, Basel, Switzerland). All devices were maintained according to standards established by the hospital's department of pathology. For the POC devices, control checks were performed by qualified anesthesia technicians at least every 24 hours, and anesthesia care providers received initial and periodic training including satisfactory completion of a multiple choice test covering proper device operation. Yearly satisfactory performance of test measurements using a high and low control sample was required to maintain certification. Providers not certified were blocked from performance of POC devices by software installed on the devices.

Glucose determinations, the time of measurement, and the manually entered patient medical record number were transmitted over the internal hospital network to a central database when POC devices were docked in the charging station. 


\section{Results}

Of the 17 patients who experienced a hypoglycemic event, eight of them were likely preventable, with another two being possibly preventable (Table 1). The four most common causes were ineffective communication, circulatory shock, failure to monitor, and excessive insulin administration (Table 2).

\section{$\underline{\text { Most Common Root Causes }}$}

Ineffective Communication - Patients 1, 2, 9, 10, 13, 15, and 16

Inadequate communication was at least partially responsible for seven hypoglycemic episodes (Table 1). Verbal instructions to hold the morning insulin in Patient 1 in the preoperative testing clinic were ineffective in achieving the desired goal. For the other patients, there was no communication between the patient's medical team and the anesthesiology providers regarding a preoperative hypoglycemic incident. In all six cases, a potentially avoidable delay occurred in the measurement of the first intraoperative $\mathrm{BG}$, each of which was in the hypoglycemic range.

Circulatory Shock - Patients 9, 11, 12, 13, 14, and 17

The cause of hypoglycemia was either septic (5) or cardiogenic (1) shock (Table 1). Although case details differed, these patients experienced similar events and hospital courses. Only two out of the six were diabetic and all required emergency surgery and were American Society of Anesthesiologists Physical Status 4E or 5. Five of the six patients died from their underlying disease within the first 10 postoperative days. 
Failure to Monitor-Patients 2, 3, 4, 6, and 15

Patient 6 had no BG checked for over three hours while on an infusion prior to surgery; the first intraoperative BG revealed hypoglycemia. Patient 4 was at risk for hypoglycemia based on a large insulin dose administered that morning while he was NPO, and there was a failure to monitor and to follow up after treatment of hypoglycemia for over six hours. Patient 15 experienced a failure to recheck BG for over 90 minutes after treatment for hypoglycemia. Although Patient 3 received a reasonable insulin bolus dose (regular insulin 2 units) shortly after entering the OR for an elevated BG measured upon hospital arrival, her known history of brittle diabetes should have prompted a repeat BG in the OR before any insulin was given. Despite the differing clinical scenarios for Patients 3, 4, and 15, all three warranted a repeat BG after entering the OR. Patient 2 had no BG checked for 12 hours while NPO after receiving a large insulin dose.

Excessive Insulin Administration - Patients 1, 2, 4, and 16

These patients received an excessive dose of insulin in the immediate preoperative period. Patient 1 took a normal insulin dose at home while NPO, while Patients 2, 4, and 16 all were given large doses of insulin as inpatients while NPO for surgery. Inadequate BG monitoring for Patients 2 and 4 contributed to their hypoglycemic episodes. 


\section{Discussion}

Our results indicate that recognized hypoglycemia was rare in this large surgical population. However, nearly half of the cases involved preventable provider errors, often caused by ineffective communication. Failure to follow established hospital protocols for BG monitoring contributed to hypoglycemia in approximately a third of patients. A third of the hypoglycemic events occurred in critically ill patients in circulatory shock.

Several hypoglycemic events occurred during the transition from medical ward or ICU to the OR/holding area, a vulnerable time where appropriate communication during hand-offs is extremely important. As articulated by Amato-Vealey et al. [4], surgical patients are susceptible to hand-off errors due to the large number of checkpoints and transitions involved in their care. This is particularly germane with glycemic management, which is dynamic and dependent upon frequent monitoring to minimize complications. For ICU patients a process was added whereby the nurse taking care of the patient calls the supervising anesthesiologist to provide a complete update on the patient's clinical status, medications, and labs.

Our instructions to patients to hold insulin on the morning of surgery are consistent with recommendations from the Society for Ambulatory Anesthesia [5]. Instructions preferably should be written, rather than verbal, to avoid potential confusion. A reminder regarding insulin instructions repeated at the time of the preoperative phone call the evening before surgery might be useful.

Hypoglycemia in patients in shock represents an entity distinct from iatrogenic hypoglycemia. Studies of ICU patients have found an association between hypoglycemia and both sepsis and circulatory shock [6,7]. All six such patients were non-diabetic, 
which reinforces the need for increased vigilance and monitoring of this group of patients, in whom metabolic derangements and hypoglycemia may be associated with increased mortality [8]. It is not clear what role these events played in the hospital course of these patients whose expected mortality would have been very high. Hypoglycemia might be a marker of poor outcome in shock rather than a cause.

Although elimination of intraoperative hypoglycemia is desirable, this goal might be difficult to achieve. In our series, one patient had an anaphylactic reaction to thymoglobulin, which has been described in case reports but not previously associated with hypoglycemia $[9,10]$. Another patient whose preoperative BG was normal had an unexpected hypoglycemic event, despite not taking any insulin or oral medications the morning of surgery.

Our study has several limitations. Manual entry of POC glucose measurements could have led to documentation errors. This was addressed by confirmation of all hypoglycemic POC BGs with values in the central POC database, resulting in the identification of one spurious value. Our prevalence of hypoglycemia underestimates the true incidence because sampling was intermittent and not performed in all patients. Prospective studies with higher sampling frequencies or continuous glucose monitoring would be required to determine the extent to which unrecognized intraoperative hypoglycemia occurs and its consequences.

Another limitation is that, in most cases, hypoglycemia identified by POC testing was not confirmed by a central lab measurement or a repeat POC determination. Given the known inaccuracy of POC glucose meters [3], some of the hypoglycemic events 
identified may have been spurious. Also, some POC values $>40 \mathrm{mg} / \mathrm{dL}$ might actually have been in the hypoglycemia range.

A final limitation of the study is that in a few cases, documentation in the chart was inadequate to identify a root cause of the hypoglycemic event.

In conclusion, hypoglycemia was detected rarely in this large surgical population, but was mostly preventable. Human factors played a role in many of the cases. The occurrence of hypoglycemia in non-diabetic, critically ill patients in shock suggests a need for close BG monitoring in this patient group. Better communication with the anesthesia care team regarding preoperative hypoglycemic events is especially important to provide appropriate follow-up and additional treatment, as necessary. 


\section{References}

1. Leese GP, Savage MW, Chattington PD, Vora JP. The diabetic patient with hypertension. Postgrad Med J 1996;72:263-268

2. Akhtar S, Barash PG, Inzucchi SE. Scientific principles and clinical implications of perioperative glucose regulation and control. Anesth Analg 2010;110:478-97

3. Rice MJ, Pitkin AD, Coursin DB. Glucose Measurement in the Operating Room: More Complicated than It Seems. Anesth Analg 2010;110:1056-63

4. Amato-Vealey EJ, Barba MP, Vealey RJ. Hand-Off Communication: A Requisite for Perioperative Patient Safety. AORN 2008;88;763-74

5. Joshi GP, Chung F, Vann MA, et al. Society for Ambulatory Anesthesia Consensus Statement on Perioperative Blood Glucose Management in Diabetic Patients Undergoing Ambulatory Surgery. Anesth Analg 2010;111:1378-87

6. Arabi YM, Tamim HM, Rishu AH. Hypoglycemia with intensive insulin therapy in critically ill patients: Predisposing factors and association with mortality. Crit Care Med $2009 ; 37: 2536-44$

7. Vriesendorp TM, van Santen S, DeVries JH, et al. Predisposing factors for hypoglycemia in the intensive care unit. Crit Care Med 2006;34:96-101

8. Krinsley JS, Grover A. Severe hypoglycemia in critically ill patients: Risk factors and outcomes. Crit Care Med 2007;35:2262-7

9. Loushin MK, Hasinoff IK, Belani KG. A Delayed Cardiopulmonary Reaction to an Intravenous Immunosuppressant Thymoglobulin After Pancreas Transplant. Anesth Analg 2001;93:1260-1 
10. Busani S, Rinaldi L, Begliomini B, Pasetto A, Girardis M. Thymoglobulin-induced severe cardiovascular reaction and acute renal failure in a patient scheduled for orthotopic liver transplantation. Minerva Anestesiol 2006;72:243-8 